\title{
Neurocutaneous Melanosis Associated With Malignant Leptomeningeal Melanoma in an Adult: Clinical Significance of 5-S-Cysteinyldopa in the Cerebrospinal Fluid
} -Case Report-

\author{
Kiyohito Shinno, Shinji NAGAhIRO, Masaaki UnO, Seiji KANNUKI, \\ Mami NAKAISO, Nobuya SANO*, and Hidehisa HORIGUCHI*
}

Departments of Neurological Surgery and *Pathology, The University of Tokushima, Tokushima

\begin{abstract}
A 35-year-old male presented with a variant of neurocutaneous melanosis with leptomeningeal malignant melanoma. He had three pigmented nevi from birth. He suffered diplopia followed by headache. $\mathrm{T}_{1}$-weighted magnetic resonance (MR) imaging revealed hydrocephalus and a small linear hyperintense lesion in the right frontal cortex. Several parts of the cortical sulci and the brain surface were slightly enhanced by gadolinium. Ventriculoperitoneal shunting was performed and extensive pigmented leptomeninges were recognized. Open biopsy established the diagnosis of leptomeningeal malignant melanoma. Combined chemoimmunotherapy was repeated every other month with monitoring of the 5-S-cysteinyldopa (5-S-CD) level in the cerebrospinal fluid (CSF). The 5-S-CD level decreased after each treatment, but the basal level steadily increased prior to the next treatment. Two years after the onset, he showed paraplegia caused by an extramedullary mass at the T-6 level. MR imaging showed that melanoma had involved the entire subarachnoid space including the whole spine. He underwent emergent removal of the spinal tumor and showed transient marked improvement. Further intensive chemotherapy was given. However, he died 31 months after the onset of massive proliferation of intracranial leptomeningeal melanoma. Measurement of CSF 5-S-CD levels is valuable for evaluating the therapeutic efficacy and for monitoring the progression of melanoma.
\end{abstract}

Key words: 5-S-cysteinyldopa, hydrocephalus, leptomeninges, melanoma, pigmented nevus

\section{Introduction}

The association of giant or multiple congenital nevi and leptomeningeal melanosis is uncommon but well documented..$^{6,20,21)}$ Leptomeningeal melanosis associated with large but not giant congenital pigmented nevi has been classified as formes frustes of neurocutaneous melanosis (NCM). ${ }^{3}$ NCM is a rare disorder involving the skin and central nervous system (CNS), and is characterized by giant or multiple cutaneous pigmented nevi (with a diameter $>20 \mathrm{~cm}$ or at least 3 lesions) present from birth. Melanosis of the leptomeninges may be present but not primary malignant melanoma in any organ other than the CNS, and no malignant change in any skin lesions. ${ }^{6,14)}$ Hydrocephalus is common, probably due to the diffuse infiltration of melanocytes into the leptomeninges at the base of the brain or the arachnoid villi. ${ }^{6,12,20)}$

Premortem diagnosis of leptomeningeal melanoma was difficult before magnetic resonance (MR) imaging with gadolinium allowed the detection of leptomeningeal involvement. ${ }^{2)}$ The prognosis for patients with NCM is extremely poor and no effective method is available to monitor the clinical course. ${ }^{5,10,15,22,25)}$

We treated a patient with variant NCM and associated malignant leptomeningeal melanoma with combined chemoimmunotherapy and followed up using the cerebrospinal fluid (CSF) 5-S-cysteinyl-

Received March 4, 2003; Accepted June 27, 2003

Author's present address: K. Shinno, M.D., Department of Neurosurgery, National Kochi Hospital, Kochi, Japan. 


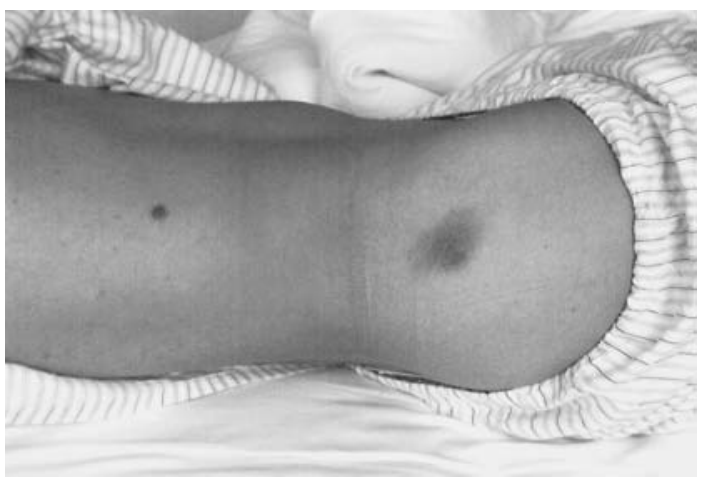

Fig. 1 Photograph of the patient showing large and small pigmented nevi on the right lateral back and hip.

dopa (5-S-CD) level.

\section{Case Report}

A 35-year-old man had two small nevi and one large blackish-brown pigmented nevus on his right lateral back, hip, and left thigh from birth (Fig. 1). He suddenly experienced diplopia followed by throbbing headache on March 27, 1997. Ophthalmological examination identified marked bilateral papilledema. He was referred to our hospital for further examination on May 11, 1997.

Neurological examination on admission revealed diplopia on left lateral gaze. The opening pressure at lumbar puncture was $390 \mathrm{~mm} \mathrm{H}_{2} \mathrm{O}$. Laboratory examination of the CSF found normal chemistry values and normal cell count. $\mathrm{T}_{1}$-weighted $\mathrm{MR}$ imaging demonstrated mild ventriculomegaly and a small linear high intensity lesion in the right frontal cortex (Fig. 2). MR imaging with gadolinium revealed linear high-intensity lesions along the brain surface and sulci (Fig. 3). Right carotid angiography showed poor filling of the superior sagittal sinus and collateral circulation via the interdiploic vein. ${ }^{111}$ Indium-diethylenetriaminepenta-acetic acid cisternography revealed both ventricular reflux and delayed clearance of the radioisotope.

Although the cause of the hydrocephalus was unknown, a ventriculoperitoneal shunt was placed using a HAKIM $^{\mathrm{TM}}$ programmable valve shunt system (Johnson \& Johnson Company, Medos S.A., Le Locle, Switzerland). The leptomeninges at the burr hole showed diffuse blackish change. Biopsy of the leptomeninges revealed leptomeningeal melanocytic proliferation (Fig. 4). Histological examination of the cutaneous lesions found benign melanocytic nevi. Eighteen days after placing the ventriculoperitoneal shunt, an open biopsy was per-
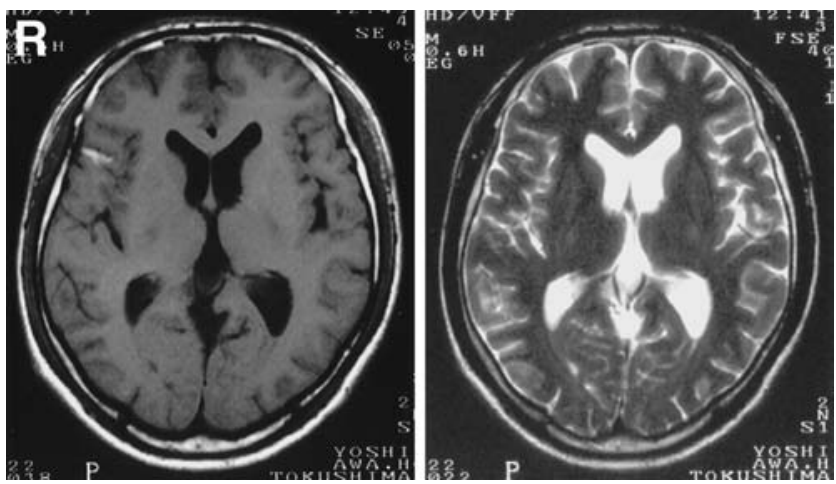

Fig. 2 Axial $\mathrm{T}_{1}$-weighted magnetic resonance (MR) image of the brain (left) showing a linear hyperintense lesion, and axial $T_{2}$-weighted MR image (right) showing the isointense lesion in the right frontal cortex.

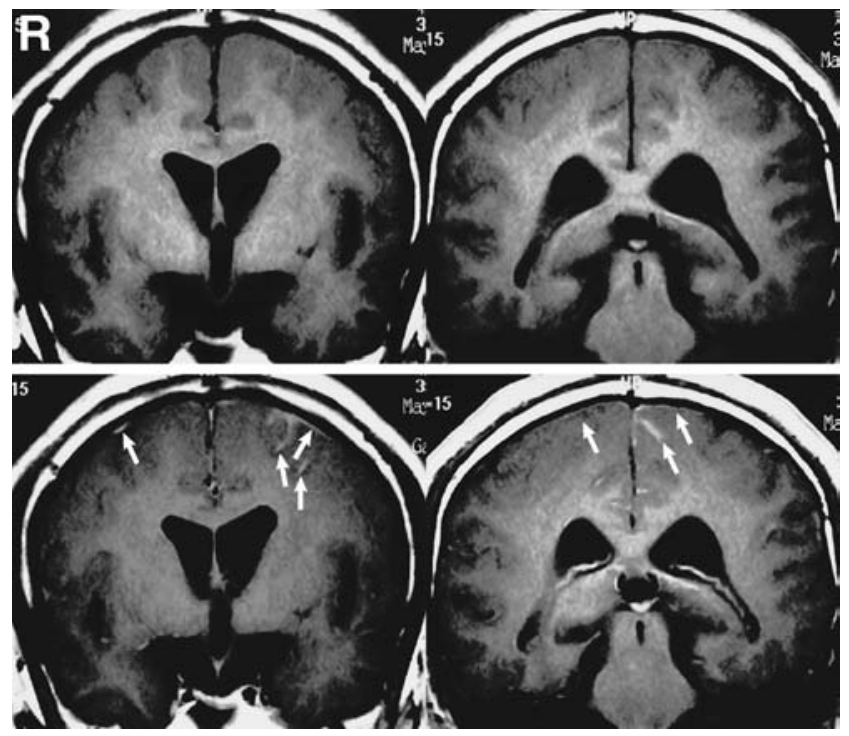

Fig. 3 Coronal $T_{1}$-weighted magnetic resonance images of the brain before (upper row) and after (lower row) gadolinium administration demonstrating linear hyperintense lesions along the cortical sulci and the brain surface (arrows).

formed in the right frontal region, where MR imaging had shown a linear high-intensity lesion, to explore for the presence of malignant transformation. The entire brain surface in the right frontotemporal region was covered by blackish leptomeninges (Fig. 5). An in-line shunt filter was placed to prevent dissemination of the malignant cells.

Histological examination of the leptomeningeal tissue revealed diffuse proliferation of melanincontaining cells. The oval, polygonal cells had a 


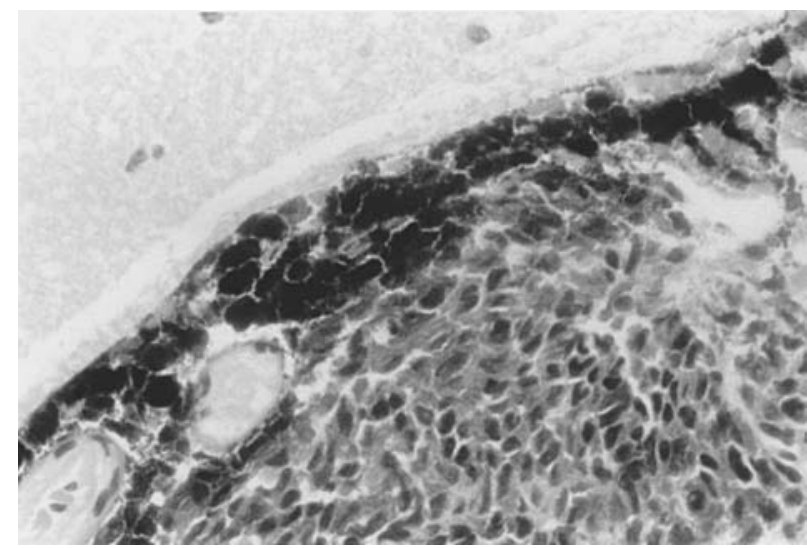

Fig. 4 Photomicrograph of a leptomeningeal sample showing proliferation of melanocytic nevus cells. HE stain, $\times 400$.

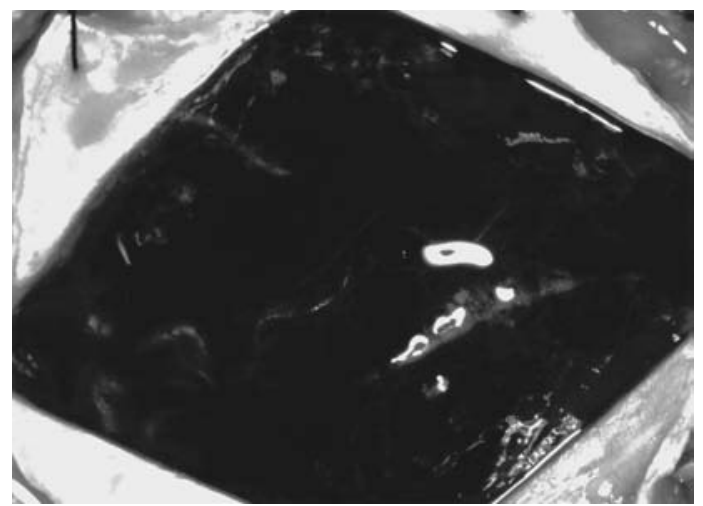

Fig. 5 Intraoperative photograph of the right frontotemporal craniotomy site revealing extensive blackish leptomeninges.

high nucleus-to-cytoplasm ratio, with irregularly contoured nuclei and prominent nucleoli (Fig. 6). HMB-45 immunohistochemistry also showed proliferation of melanin-containing cells (Fig. 7). The cells had infiltrated the Virchow-Robin space. The MIB-1 labeling index was $11.0 \%$. The histological diagnosis was malignant leptomeningeal melanoma.

Postoperative computed tomography of the chest, abdomen, and pelvis showed no evidence of lymphadenopathy. The patient received six courses of chemotherapy consisting of cisplatin $\left(60 \mathrm{mg} / \mathrm{m}^{2}\right.$, first day), dacarbazine ( $80 \mathrm{mg} / \mathrm{m}^{2}$, 2nd to 6th days), and vindesine $\left(2 \mathrm{mg} / \mathrm{m}^{2}\right.$, 2nd day) followed by interferon $-\beta\left(300 \times 10^{4} \mathrm{U} /\right.$ day, 7 th to 9 th days $)$ every other month. The levels of 5-S-CD, a catechol metabolite of dihydroxyphenylalanine and a marker for melanocytes, were measured in both serum and

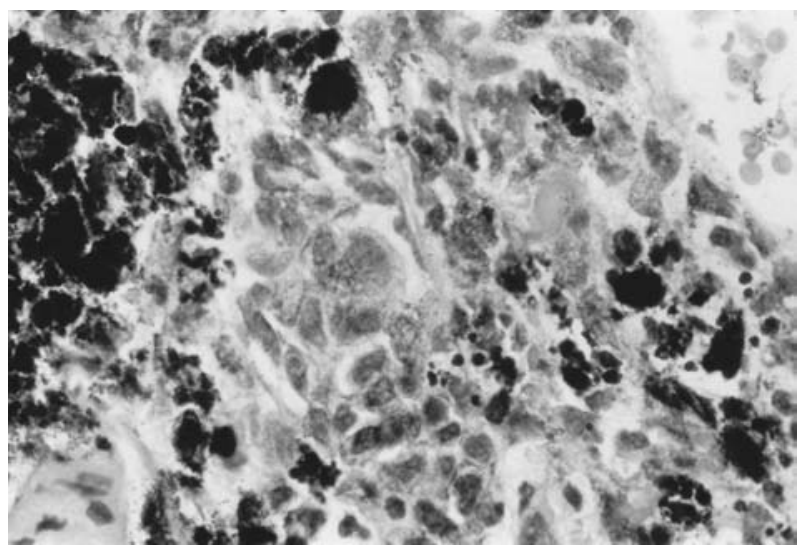

Fig. 6 Photomicrograph of the leptomeningeal melanin-containing tumor showing both nucleolar and cellular pleomorphism with a markedly high nucleus-to-cytoplasm ratio and prominent nucleoli. HE stain, $\times 400$.

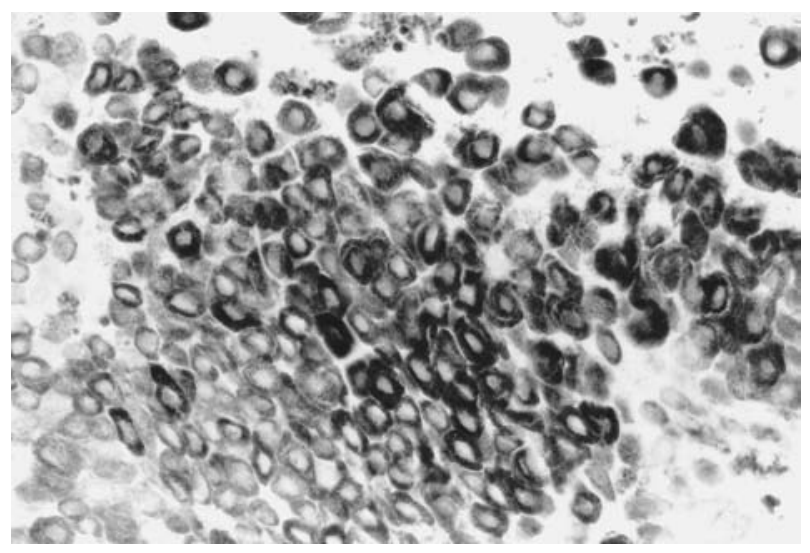

Fig. 7 Photomicrograph of the leptomeningeal tumor showing proliferation of melanincontaining cells. HMB-45 immunohistochemistry, $\times 400$.

CSF before and after each treatment course. The CSF was sampled from the chamber of the shunt system. The initial value of CSF $5-S-C D$ was $71.0 \mathrm{nmol} / \mathrm{l}$; the serum 5-S-CD level was normal at $4.9 \mathrm{nmol} / \mathrm{l}$ (normal range 1.5-8.0 nmol/l). The CSF level of 5-SCD decreased after each course of chemotherapy (Fig. 8). There was a steady increase in the basal level of CSF 5-S-CD prior to the treatment. The serum 5-S-CD value was consistently normal for at least one year.

One year after the onset of symptoms, the patient remained neurologically normal. MR imaging showed no progression of the right sylvian lesion, but gyral enhancement of the left frontal cortex 


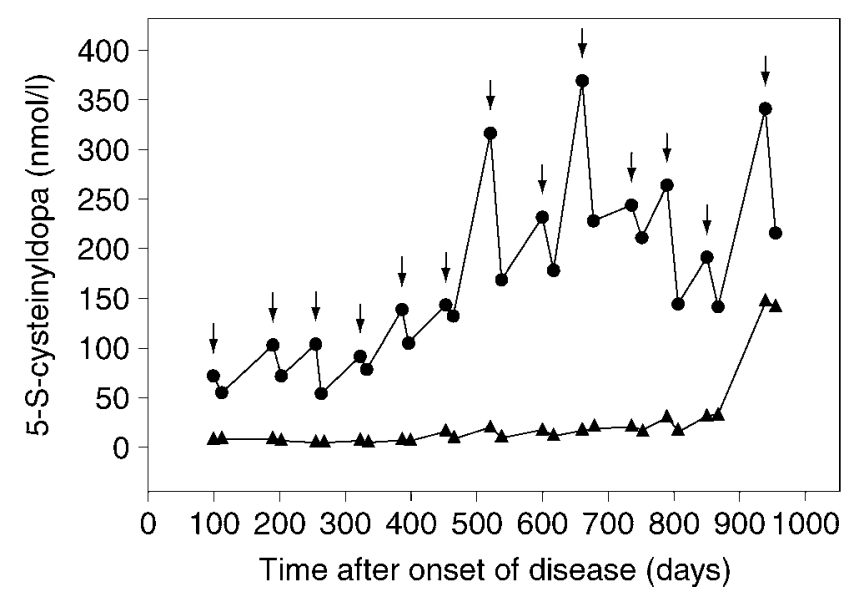

Fig. 8 Changes in 5-S-cysteinyldopa levels in the serum (closed triangles) and cerebrospinal fluid (closed circles) before and after chemoimmunotherapy using cisplatin, dacarbazine, vindesine, and interferon- $\beta$. Arrows indicate each course of chemoimmunotherapy.

increased slightly. CSF cytology revealed the presence of malignant cells. Spinal $\mathrm{T}_{1}$-weighted $\mathrm{MR}$ imaging revealed extensive hyperintense involvement of the subarachnoid space. Therefore, five more courses of intensive chemoimmunotherapy were given consisting of cisplatin $\left(125 \mathrm{mg} / \mathrm{m}^{2}\right.$, first day), dacarbazine (200 mg/m $\mathrm{m}^{2}$, 2nd to 6 th days), and vindesine $\left(4.5 \mathrm{mg} / \mathrm{m}^{2}\right.$, 2nd day) followed by interferon- $\beta\left(300 \times 10^{4} \mathrm{U} /\right.$ day, 7 th to 16 th days $)$ every other month. However, the CSF 5-S-CD levels gradually showed further deterioration (Fig. 8). The patient began to show generalized convulsion once a month in spite of increased doses of anticonvulsants 18 months after the onset of symptoms. MR imaging showed that the entire subarachnoid space was extensively involved with melanoma 2 years after the onset (Fig. 9).

The patient was doing well without neurological deficit until April 1999, when he showed gradual progression of paraplegia and sensory disturbance in the lower trunk and legs 25 months after the onset. He was readmitted to our hospital on May 10, 1999. $\mathrm{T}_{1}$-weighted $\mathrm{MR}$ imaging revealed extensive hyperintense involvement of the subarachnoid space and a solitary extramedullary round mass compressing the spinal cord at the T-6 level (Fig. 10 left).

Emergent removal of the tumor was performed. Laminectomy of the T-5 to T-7 levels showed that the entire subarachnoid space was covered with blackish leptomeningeal melanoma, like the brain sur-

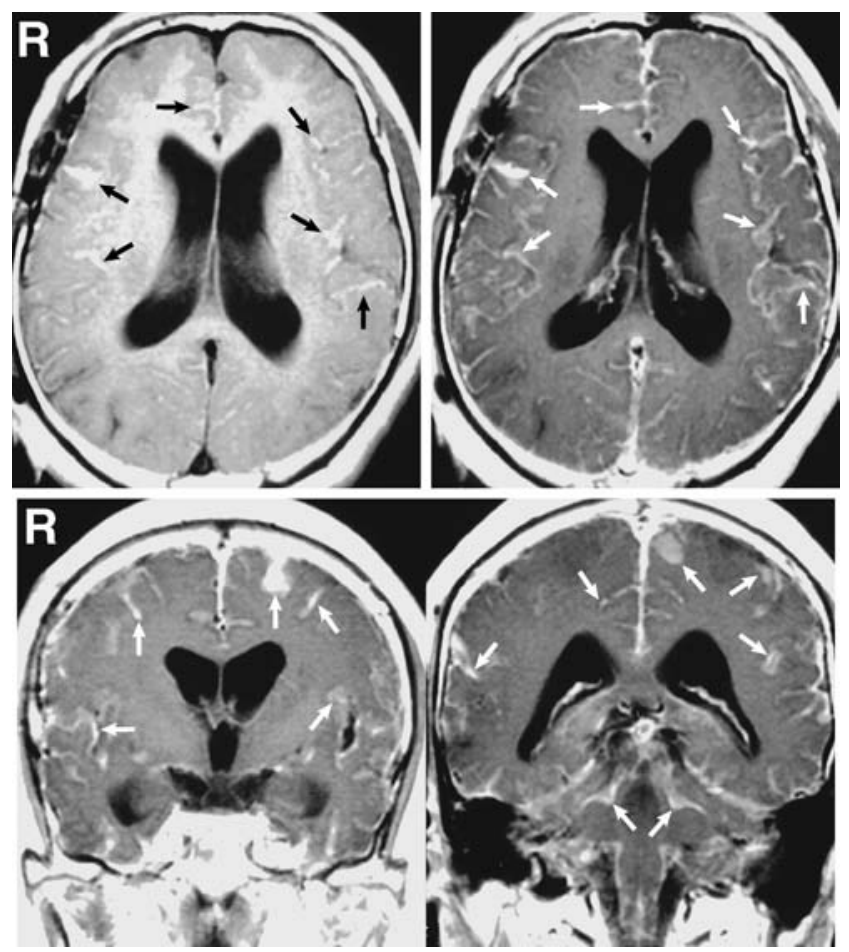

Fig. 9 Magnetic resonance (MR) images 2 years after the onset showing diffuse involvement of subarachnoid space with melanoma. Axial $T_{1}$-weighted $M R$ image (upper left) showing slightly hyperintense extensive lesions occupying the subarachnoid space. Axial (upper right) and coronal (lower row) $T_{1}$-weighted MR images after gadolinium administration showing marked involvement of the entire subarachnoid space with enhanced lesions. Obvious progression of melanoma has occurred in the cortical sulci compared with the initial findings (arrows).

face. The tumor was located using intraoperative B-mode ultrasonography and subtotally removed. Postoperative MR imaging revealed recovery of the spinal compression and absence of mass lesion (Fig. 10 right).

Histological examination of the spinal tumor revealed diffuse proliferation of melanin-containing polygonal cells with irregularly contoured nuclei and prominent nucleoli. HMB-45 immunohistochemistry showed proliferation of melanin-containing cells (Fig. 11). The MIB-1 labeling index was 3.0\%. The diagnosis was malignant melanoma.

The patient showed marked improvement of the weakness postoperatively and started rehabilitation. However, he began to suffer frequent generalized convulsion from June 1999. Brain MR imaging revealed massive proliferation of the leptomeningeal 

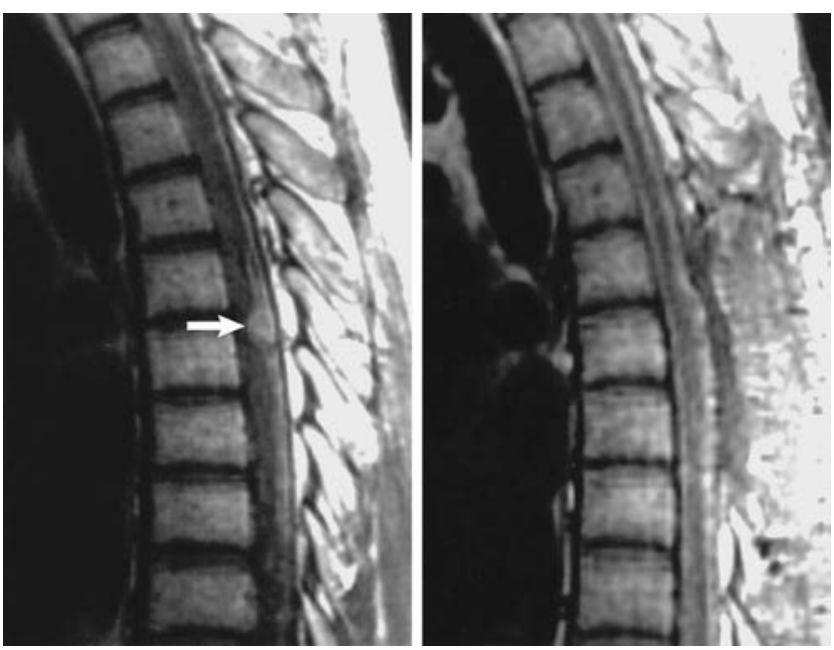

Fig. 10 Sagittal $T_{1}$-weighted magnetic resonance (MR) image of the thoracic spine (left) taken 25 months after the onset demonstrating linear hyperintense lesions along the subarachnoid space of the spinal cord and a solitary hyperintense round mass at the T-6 level (arrow). Postoperative sagittal $T_{1}$-weighted MR image (right) showing no mass lesion at the same level.

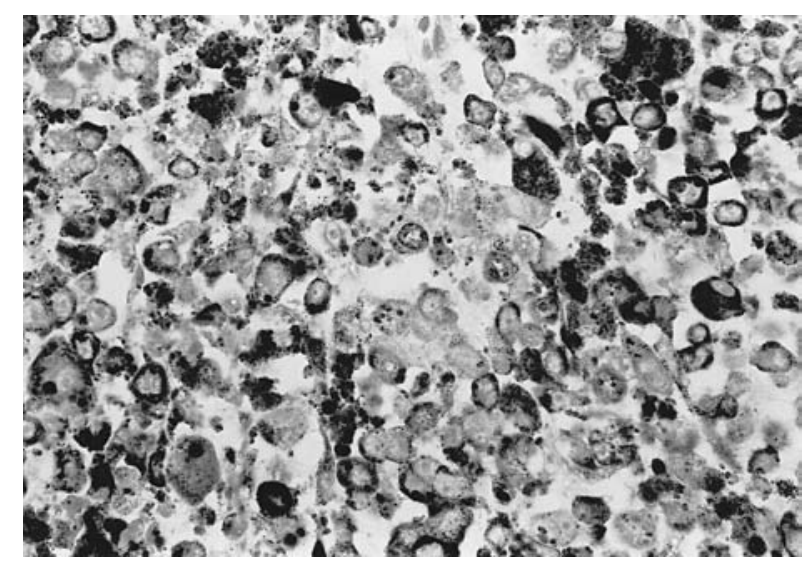

Fig. 11 Photomicrograph of the extramedullary pigmented spinal tumor revealing proliferation of melanin-containing cells with nucleolar and cellular pleomorphism. HMB-45 immunohistochemistry, $\times 400$.

melanoma with nodular enlargement. Two courses of intensive chemoimmunotherapy were given, but he gradually showed motor aphasia and tetraparesis followed by drowsiness. He died of marked enlargement of intracranial melanoma on November 4, 1999. Postmortem examination disclosed extensive involvement of the entire subarachnoid space of the CNS by malignant melanoma. Intraperitoneal dissemination of melanoma via the ventriculoperitoneal shunt was also recognized.

\section{Discussion}

The present patient probably had a variant of NCM because the congenital nevi together measured less than the minimum required by the diagnostic criteria. ${ }^{6)}$ Our case of NCM is difficult to differentiate strictly from primary leptomeningeal melanoma with large congenital nevi, ${ }^{10)}$ as both disorders frequently manifest similar clinical features, and may share a similar pathogenesis.

Histological confirmation is essential for a confident diagnosis of NCM, but leptomeningeal melanoma can be visualized by MR imaging because of the unusual paramagnetic properties of melanin. ${ }^{23)}$ Intracranial melanomas typically appear bright on $\mathrm{T}_{1}$ - and dark on $\mathrm{T}_{2}$-weighted images ${ }^{26)}$ because of the presence of free paramagnetic radicals from melanin which reduce the relaxation times. ${ }^{23)}$ We recognized a linear high-intensity lesion along the cortical sulcus on the $\mathrm{T}_{1}$-weighted images and gadolinium enhancement demonstrated diffuse leptomeningeal involvement on both the brain surface and the cortical sulci. These observations suggest that careful attention must be paid to changes along the brain surface, especially after gadolinium enhancement, to obtain the correct diagnosis prior to histological examination.

The leptomeningeal lesions associated with NCM usually undergo malignant transformation ${ }^{6,15)}$ and the prognosis is extremely poor. Almost all patients with malignant transformation survived for less than one year ${ }^{5,10,15,17,22,25)}$ because currently no effective treatment is available. ${ }^{14)}$ Malignant melanoma is one of the most radioresistant tumors ${ }^{7,15)}$ and no beneficial chemotherapy has been developed for NCM..$^{5,10,17)}$ However, dacarbazine combined with radiotherapy was effective in one patient with a recurrent solitary leptomeningeal melanoma. ${ }^{16)}$ Chemoimmunotherapy using dacarbazine and vincristine with OK432 was effective in the treatment of metastatic malignant melanoma of the brain. $\left.{ }^{4}\right)$ Therefore, we administered dacarbazine, the most common chemotherapeutic agent currently used for melanoma, in combination with cisplatin, vindesine, and interferon- $\beta$ every other month according to the treatment regimen for advanced malignant melanoma. ${ }^{18,24)}$ Eleven courses of this chemoimmunotherapy were administered. Our patient manifested no further clinical deterioration until 2 years after the onset of symptoms. However, MR imaging showed that the leptomeningeal tumor of 
the CNS including entire spinal cord had obviously progressed and a solitary pigmented mass lesion was recognized at the T-6 level 25 months after the onset. Since he finally died of massive proliferation of the intracranial leptomeningeal melanoma 31 months after the onset, this therapeutic regimen seems to have a definite but limited effect on NCM.

The levels of 5-S-CD, a catechol metabolite of dihydroxyphenylalanine, a precursor of pheomelanin, and a known marker for melanocytes, ${ }^{1,8,9,11,13)}$ were measured in both the serum and CSF before and after each treatment course in our patient. The importance of periodically measuring serum and urinary 5-S-CD as a biochemical marker for melanoma progression, for determining the stage, detecting metastases, and assessing the therapeutic efficacy of surgery or chemoimmunotherapy has been described in patients with malignant melanoma. ${ }^{9,11}$, 13,19) However, CSF levels of 5-S-CD have not been monitored in patients with NCM, so the basal 5-SCD CSF levels were unclear. Therefore, we measured 5-S-CD in CSF collected from 10 patients with Parkinson disease $(n=5)$, hemifacial spasm $(n=3)$, and moyamoya disease $(n=2)$ during surgery. The basal 5-S-CD CSF level was determined as $0 \mathrm{nmol} / \mathrm{l}$. In our patient, the basal level of 5-S-CD in the CSF was significantly elevated.

The 5-S-CD CSF level decreased after each treatment, but the pretreatment level tended to increase gradually during the course. The serum level of 5-SCD was consistently normal or much lower than the CSF level for 2 years after the onset, so we think that no melanoma was present outside the CNS. However, the basal level of 5-S-CD in the CSF continued to increase despite the treatment, so we suspect that subclinical progression of the leptomeningeal melanoma of the CNS occurred. The progression of melanoma of the brain may have caused the gradual increase in seizures. A solitary pigmented mass of the spinal cord was discovered 2 years after the onset.

Our findings suggest that periodic measurement of CSF 5-S-CD is valuable for evaluating the therapeutic efficacy of chemoimmunotherapy in patients with malignant leptomeningeal melanoma and for monitoring the progression of the melanoma.

\section{References}

1) Agrup G, Andersson T, Falck B, Persson K, Rorsman H, Rosengren A-M, Rosengren E: 5-S-cysteinyldopa in the plasma of melanoma patients and the renal clearance of this amino acid. Acta Derm Venereol 55: 5-6, 1975
2) Allcutt D, Michowiz S, Weitzman S, Becker L, Blaser S, Hoffman HJ, Humphreys RP, Drake JM, Rutka JT: Primary leptomeningeal melanoma: An unusually aggressive tumor in childhood. Neurosurgery 32: 721-729, 1993

3) Bamborschke S, Ebhardt G, Szelies-Stock B, Dreesbach HA, Heiss W-D: Review and case report: Primary melanoblastosis of the leptomeninges. Clin Neuropathol 4: 47-55, 1985

4) Chiba M, Jimbow K, Kizukuri K, Homma K: Chemoimmunotherapy for disseminated malignant melanoma with DTIC, ACNU, VCR and OK432. J Dermatol 9: 23-30, 1982

5) Faillance WJ, Okawara S-H, McDonald JV: Neurocutaneous melanosis with extensive intracerebral and spinal cord involvement. J Neurosurg 61: 782-785, 1984

6) Fox H: Neurocutaneous melanosis, in Vinken PJ, Bruyn GW (eds): The Phakomatoses. Handbook of Clinical Neurology, vol 14. Amsterdam, Elsevier, 1972, pp 414-428

7) Habermalz HJ, Fischer JJ: Radiation therapy of malignant melanoma - Experience with high individual treatment doses. Cancer 38: 2258-2262, 1976

8) Hansson C, Agrup G, Rorsman H, Rosengren A-M, Rosengren E: An analysis of cysteinyldopas, dopa, dopamine, noradrenaline and adrenaline in serum and urine using high-performance liquid chromatography and electrochemical detection. $J$ Chromatogr 162: 7-22, 1979

9) Hara H, Chino K, Kawanami $T$, Sameshima $T$, Morishima T: 5-S-cysteinyldopa in urine and tumors. J Dermatol 19: 806-808, 1992

10) Hoffman HJ, Freeman A: Primary malignant leptomeningeal melanoma in association with giant hairy nevi. J Neurosurg 26: 62-71, 1967

11) Horikoshi T, Ito S: Serum 5-S-cysteinyldopa (5-S-CD) as a marker of melanoma progression. J Dermatol 19: 809-813, 1992

12) Humes RA, Roskamp J, Eisenbrey AB: Melanosis and hydrocephalus. J Neurosurg 61: 365-368, 1984

13) Jönsson P-E, Agrup G, Arnbjörnsson E, et Hafström LO, Rorsman H: Treatment of malignant melanoma with dacarbazin (DTIC-DOME) with special reference to urinary excretion of 5-S-cysteinyldopa. Cancer 45: 245-248, 1980

14) Kadonaga JN, Frieden IJ: Neurocutaneous melanosis: Definition and review of the literature. J Am Acad Dermatol 24: 747-755, 1991

15) Kaplan AM, Itabashi HH, Hanelin LG, Lu AT: Neurocutaneous melanosis with malignant leptomeningeal melanoma. Arch Neurol 32: 669-671, 1975

16) Nakagawa H, Hayakawa $T$, Niiyama $K$, Nii $Y$, Yoshimine T, Mori S: Long-term survival after removal of primary intracranial malignant melanoma. Case report. Acta Neurochir (Wien) 101: 84-88, 1989

17) Pascual-Castroviejo I: Neurocutaneous melanosis, in Gomez M (ed): Neurocutaneous Diseases. Boston, 
Butterworth, 1987, pp 329-334

18) Pectasides D, Yianniotis H, Alevizakos N, Bafaloukos D, Barbounis V, Varthalitis J, Dimitriadis M, Athanassiou A: Treatment of metastatic malignant melanoma with dacarbazine, vindesine and cisplatin. Br J Cancer 60: 627-629, 1989

19) Peterson LL, Woodward WR, Fletcher WS, Palmquist M, Tucker MA, Ilias A: Plasma 5-S-cysteinyldopa differentiates patients with primary and metastatic melanoma from patients with dysplastic nevus syndrome and normal subjects. J Am Acad Dermatol 19: 509-515, 1988

20) Reed WB, Becker SW Sr, Becker SW Jr, Nickel WR: Giant pigmented nevi, melanoma, and leptomeningeal melanocytosis. A clinical and histopathological study. Arch Dermatol 91: 100-119, 1965

21) Slaughter JC, Hardman JM, Kempe LG, Earle KM: Neurocutaneous melanosis and leptomeningeal melanomatosis in children. Arch Pathol 88: 298-304, 1969

22) Thomas CS, Toone BK, Rose PE: Neurocutaneous melanosis and psychosis. Am J Psychiatry 145: 649-650, 1988 (letter)

23) Vasdev A, David P, Villemot D, Bennani W, Pascal-
Ortiz D, Pasquier B, Chirossel JP, Le-Bas JF: Apparently primary malignant melanoma of the cerebellopontine angle. One case. J Neuroradiol 17: 152-156, 1990

24) Verschraegen CF, Kleeberg YR, Mulder J, Rumke P, Truchetet F, Czarnetzki B, Rozencweig M, Thomas D, Suciu S: Combination of cisplatin, vindesine, and dacarbazine in advanced malignant melanoma. A phase II study of the EORTC malignant melanoma cooperative group. Cancer 62: 1061-1065, 1988

25) Williams HI: Primary malignant meningeal melanoma associated with giant hairy nevi. J Pathol Bacteriol 99: 171-174, 1969

26) Woodruff WW Jr, Djang WT, McLendon RE, Heinz ER, Voorhees DR: Intracerebral malignant melanoma: high-field-strength MR imaging. Radiology 165: 209-213, 1987

Address reprint requests to: K. Shinno, M.D., Department of Neurosurgery, National Kochi Hospital, 1-2-25 Asakura Nishi-machi, Kochi 780-8077, Japan. e-mail: sinnok@kochi2.hosp.go.jp 